\title{
Primary Cutaneous MZL with Amyloid Deposition, Initially Misdiagnosed as Amyloidosis Mimics Primary Dural MZL upon Dural Dissemination*
}

\author{
Miaoxia He ${ }^{1}$, Lili Wu ${ }^{2}$, Minghua Zhu ${ }^{1}$, Chenguang Bai ${ }^{1}$, Shimin Zhang $^{3}$, Jianjun Wang ${ }^{1 \#}$ \\ ${ }^{1}$ Department of Pathology, Changhai Hospital, Second Military Medical University, Shanghai, China; ${ }^{2}$ Department of Pathology, \\ Ruijin Hospital, Shanghai Jiao Tong University School of Medicine, Shanghai, China; ${ }^{3}$ Molecular Diagnopsis Laboratories, Armed \\ Forces Institute of Pathology, Washington DC, USA. \\ Email: \#ianjun1950@hotmail.com
}

Received September $30^{\text {th }}, 2012$; revised October $29^{\text {th }}$, 2012; accepted November $11^{\text {th }}, 2012$

\begin{abstract}
Primary cutaneous marginal zone B-cell lymphoma (MZL) is considered a cutaneous counterpart of extranodal MZL of mucosa-associated lymphoid tissue and an indolent lymphoma. Amyloid deposition in this tumor is very rare. We report here a case of primary cutaneous MZL with massive amyloid deposition. The tumor disseminated to the dura and mimicked primary dural MZL. The patient had a four year history of the recurrence cutaneous lesions with a misdiagnosis of amyloidosis. The correct diagnosis was made after the metastatic dural lesion was confirmed by radiology, pathology and molecular biology approaches. It is of crucial importance for differential diagnosis to avoid misdiagnosis and the patients are indeed required long-term regular examinations and treatment because of the possibility of recurrence or dissemination.
\end{abstract}

Keywords: Skin; Dura; Amyloid Deposition; MZL; Dissemination

\section{Introduction}

Primary cutaneous marginal zone B-cell lymphoma (MZL) is considered as a cutaneous counterpart of extranodal MZL of mucosa-associated lymphoid tissue and generally involves as indolent lymphomas [1,2]. Characteristics of cutaneous MZL include the proliferation of centrocyte-like cells or monocytoid B cells with frequent plasmacytoid differentiation. Occasionally, there were some Rusell or Dutcher bodies but rarely amyloid deposition descried in previous literature [2-5]. Here, we report a rare case of primary cutaneous MZL with massive amyloid deposition and disseminated to the dura, which mimicked the primary dural MZL.

\section{Case Report}

A 71-year-old Chinese male was admitted to the emergency room in Changhai Hospital (Shanghai, China) in June 2008 because of facial tics on the left side of his face, numbness in the left side of his body and logagnosia for 3 - 4 min. Physical examination of nervous system revealed dysesthesia on the left side of his face.

\footnotetext{
*Competing interests: the authors declare that they have no competing interests.

${ }^{\#}$ Corresponding author.
}

Computed tomography (CT) scanning of the head showed a large low density shadow in the right temporal-parietal lobe and a $6.0 \mathrm{~cm} \times 3.0 \mathrm{~cm} \times 3.0 \mathrm{~cm}$ oval high density shadow on the right temporal-parietal lobe (Figure 1(A)). Magnetic resonance imaging (MRI) scanning showed an oval occupancy on the right temporal-parietal lobe and there was a connection between tumor and the wide base of the meninges. The lesion was isointense on T1weighted and hyperintense on T2-weighted MRI images. Homogeneous contrast enhancement image mimicked a meningioma on the T1-weighted MRI (Figure 1(B)). There was marked edema throughout the posterior half of the right cerebral hemisphere (Figure 1(C)). The right ventricle was compressed and the midline was slightly left-biased. It was considered to be the right temporal parietal meningioma.

Upon the admission, a mass was found on his left para-axillary area with a diameter of $2 \mathrm{~cm}$. Also, the patient told that he had a history of a subcutaneous mass with a diameter of about $2.0 \mathrm{~cm}$ on his left elbow in 2004 and a recurrent mass $(6.0 \mathrm{~cm} \times 5.0 \mathrm{~cm} \times 4.0 \mathrm{~cm})$ on the left para-axillary area in 2007. Both were diagnosed as amyloidosis in the local county hospitals. The laboratory data and blood cell counts were within the normal ranges. The bone marrow smears showed no abnormality. No 

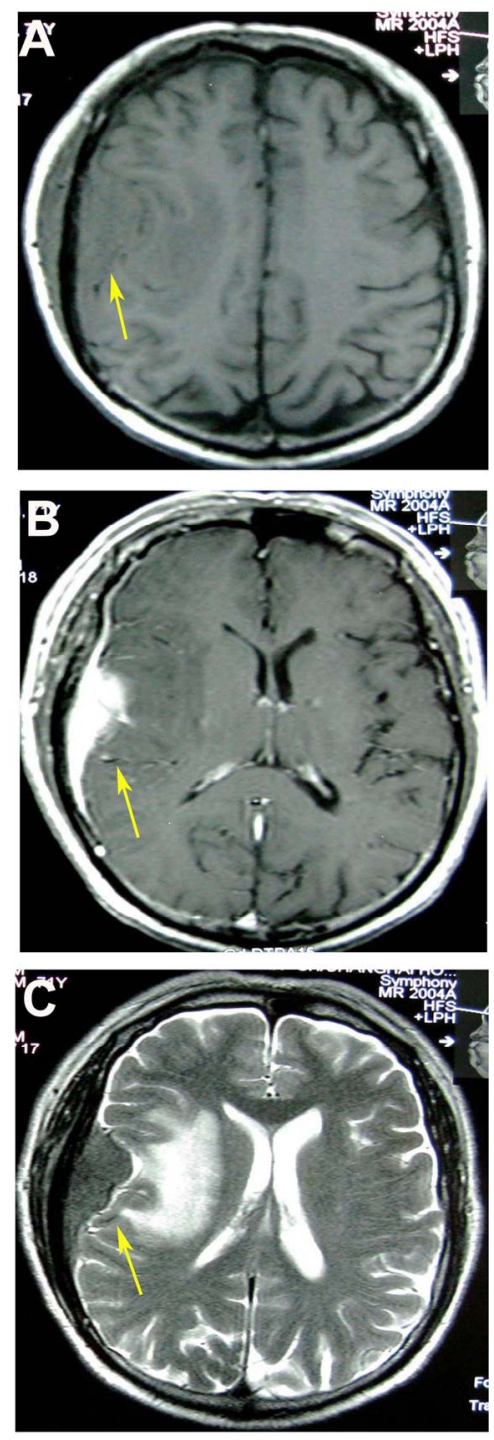

Figure 1. Radiological figures of the patient's brain. (A) Computed tomography (CT) scanning of the head showed a large lowdensity shadow in the right temporal-parietal lobe; (B) Magnetic resonance imaging (MRI) scanning of the head revealed a dura-based mass with isointense on T1weighted and hyperintense on T2-weighted MRI images. Homogeneous contrast enhancements were mimicking a meningioma on the T1-weighted magnetic resonance images; (C) There was marked edema throughout the posterior half of the right cerebral hemisphere on MRI images.

obvious abnormality was present in serum protein electrophoresis or serum immunoglobulin analysis. Results of a radiographic skeletal survey of ECT were negative. CT scanning of the chest, abdomen, and pelvis demonstrated only probable old pulmonary lesions. The preoperative cerebral edema subsided in response to a routine therapy. The patient underwent a right temporal-parietal lobe tumor resection. It was observed in the surgery that there were pinkish grey fishlike tumor tissues on the sur- face of the skull under the temporal muscle, which was adhered to the skull and the temporal muscle. The involved skull showed a local thickening and obvious moth-eaten destruction. The tumor was rich in blood supply and the surface of cerebral dural mater and subdural space were covered with tumor as soft grapelike clusters. There was a clear boundary between the surrounding of subdural tumor and brain tissues. The tumor in the rear part of the right frontal lobe invaded the brain tissue with ill-defined surroundings. Based on the neuroimaging data, the preliminary clinical impression of the disease was meningioma. The specimens were embedded in paraffin block for haematoxylin and eosin (H\&E), Congo red staining and immunostaining. Microscopically, tumor tissue was mainly located in the meninges and infiltrated to the brain tissue of the right temporal-parietal lobe. The tumor cells were small-to-medium sized with a distinct monocytoid appearance and frequent plasmacytoid/plasma cells (Figure 2(A)). There was pink amyloid substance among tumour cells aggregated to a large sheet forming amyloid pool surrounded by multinucleated giant cells (Figure 2(B)). Some foreign bodycontaining giant cells dispersed in massive amyloid deposition in the stroma, which formed part of Congo red staining positive eosinophilic amyloid pools, Congo redpositive, thickened, hyalinized blood vessels were seen in the tumor tissue (Figure 2(C)). Immunohistochemical staining revealed that most infiltrates were composed of CD20 positive B monocytoid lymphocytes (Figure 2(D)) and plasmacytoid lymphocytes which were negative for CD5, CD10 and CD23. A smaller population of CD138 positive mature plasma cells and scattered CD3 positive reactive $\mathrm{T}$ lymphocytes were also present. The vast majority of the lymphocytes and plasmacytoid cells displayed strong immunoreactivity for kappa (Figure 2(E)), but no immunoreactivity for lambda light chains, indicating a monoclonal population. The proliferative activeity determined using Ki-67 antibody was about $10 \%$. After reviewed the tissue samples of the subcutaneous masses removed from the left elbow in 2004, the left para-axillary in 2007 and the recurrence lesion in the same time of dural lesion, we found that the tumor was located in the deep dermis and subcutaneous adipose tissue, The tumor cells infiltrated to the subcutaneous fat tissue forming nodular growth pattern (Figure 2(F)), and the pinkish amyloid depositions with a blocklike distribution were seen between tumor cells. Their morphology and IHC staining patterns were similar to those of the right temporal-parietal lobe tumor. Gene rearrangement PCR results showed monoclone in the skin and brain lesions when $I G H$ primers were used (Figure 2(G)). The fluorescence in situ hybridization assay using MALT1 dual color break apart probe showed that fusion signals were present in the tissues from recurrence para-axillary 


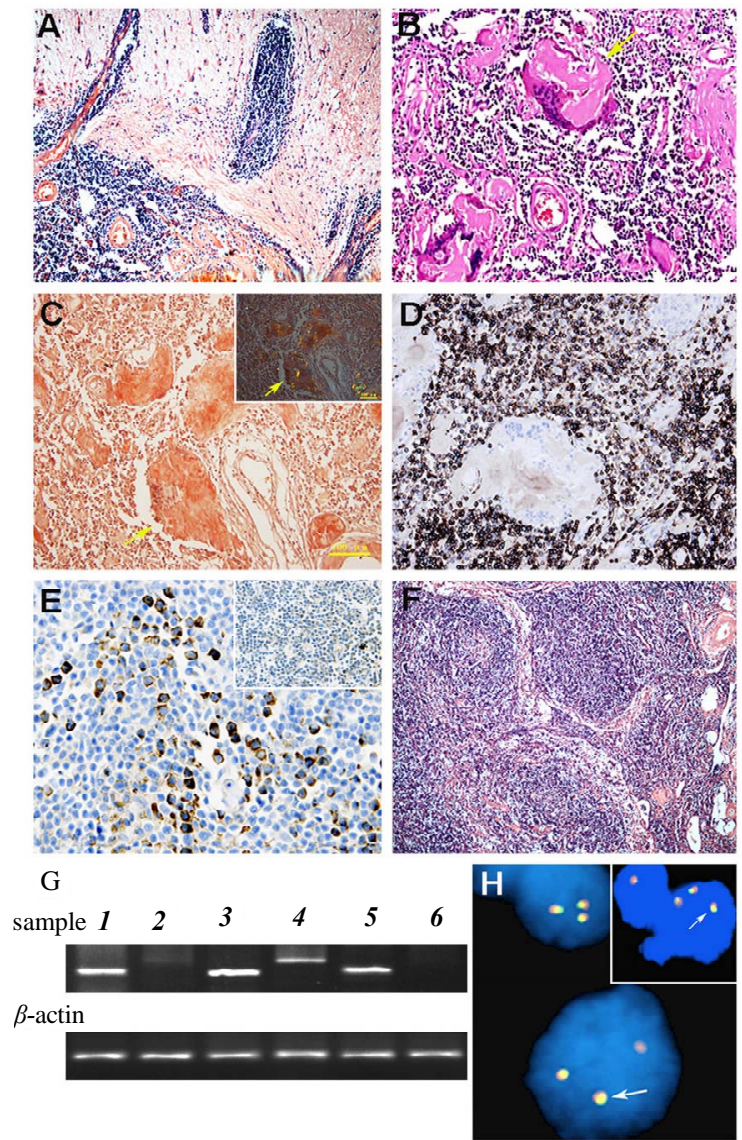

Figure 2. (A) Histologic features of dura MZLshowed lymphoid tissues extend into the cerebrum (×100); (B) Amyloid deposition within the dural lesions of MZL showed accumulation of eosinophilic amyloid pools ( HE stain $\times 200$ ). (C) Congo red stain confirmed the presence of amyloid substance of dural lesions (Congo red stain $\times 200$ with light microscope; Inserted with phase-contrast microscope). (D) immunohistochemical staining of tissue samples from the dural lesions showed the neoplastic cells were immunoreactive for B-lymphocyte marker CD20 $(\times 200)$. (E) The neoplastic cells of dural lesions were positive for immunoglobulin light kappa chain $(\times 200)$, but not for lambda chain (Inserted). (F) Histologic features of cutaeneous MZL showed lymphoid tissues infiltrate in the subcutaneous tissue forming nodular growth pattern $(\times 100)$. (G) Clonality assays of tumor cells from cutaneous and dural lesions by PCR followed by gel electrophoresis using $I G H$ and $T C R \quad \gamma$ gene: skin lesion showed monoclone band of IGH (1) but smear of TCR $\gamma$ gene (2), positve controls showed B lymphoma cell line Ly1 with monoclone band of IGH (3) and T lymphoma cell line Jurkat with monoclone band of $T C R \gamma$ (4), dural lesion aslo showed monoclone band of IGH (5) but smear of TCR $\gamma$ gene (6), PCR products for $\beta$-actin gene indicated similar DNA load in different tests. $2 \mathrm{H}$ The fluorescence in situ hybridization assay using MALT1 dual color break apart probe. Results showed that the case was negative for the translocation of MALT1 but there were three fusion signals in many tumor cells from right frontal-temporal lobe lesion $(\mathrm{H})$ and recurrence para-axillary cutaneous lesion (inserted) indicating the possibility of trisomy 18 . cutaneous lesion and right frontal-temporal lobe lesion suggesting a possibility of the existence of extra chromosome 18 (trisomy 18) (Figure 2(H)). The patient was finally diagnosed as the primary cutaneous MZL with local recurrence and relapse involving dura. The patient was given a rational whole-brain radiation therapy delivered in 20 fractions of 180 Gy for a total dose of 36 Gy and a routine chemical therapy with R-CHOP regimen. He has been followed-up at the outpatient clinic and remained free from seizures and other complains for 46 months after the brain tumor removal operation.

\section{Discussion}

The skin is one of possible locations for MZL, and the European Organization for Research and Treatment of Cancer (EORTC) Cutaneous Lymphoma Project Group has proposed cutaneous MZL as a distinct clinical pathological entity, often quite different from their typical gastrointestinal or nodal counterparts [2,5]. Rusell or Dutcher bodies were seen because of frequent plasmacytoid differentiation, but amyloid deposit was not present in most reported cases of primary cutaneous MZL [2-5]. We here report a primary cutaneous MZL with massive amyloid deposition which misdiagnosed as amyloidosis in a 71year-old male and the correct diagnosis was not established until the right temporal-parietal lobe lesion appeared four years later.

The most characteristic histological feature of this cutaneous MZL case was massive amyloid deposition in tumor tissues, which was probably the main reason for the long period misdiagnosis. Because of medium-sized lymphocytes, reactive lymphoid follicles and variable degrees of plasmacytic differentiation in the lesions, it was difficult to differentiate them from the inflammation. Meanwhile, the differentiation between the MZL and amyloidosis is very important. Amyloidosis usually presents as a systemic disease in numerous chronic immune conditions and also in some malignancies [6-8]. Localized amyloidosis is more often associated with the respiratory tract, or duct of breast $[9,10]$. Cutaneous localized amyloidosis is rare, mostly associated with plasmacyte neoplasms, or lymphoplasmacytic lymphoma exhibiting IgM monoclonal gammopathy [6,7]. Some primary dural MZLs with amyloid deposition have reported [1,11-14]. In current case, despite the same histological features and amyloid deposition as a typical primary dural MZL in the dural lesion, the patient was confirmed having the history of primary cutaneous lesion with significant amyloid deposition in multiple sites. Molecular approaches confirmed that tumor cells from the skin and dura had similar B-cell type monoclonal rearrangement of IGH gene. FISH assays also showed tumor cells from the both skin and dura lesions were chromosome 18 trisomy. There- 
fore, we conclude that his dural MZL is the secondary to the primary skin MZL.

Secondly, the significant clinical feature of this case was multiple disseminations. Although MZL is a distinctive low grade extranodal lymphoma with the indolent nature, about 50\% of patients with extra-intestinal MZL showed multi-organ involvement, compared with only about $34 \%$ of patients with gastric MALT lymphoma [15]. In a series head and neck MZL study, 52\% of patients had advanced disease and $25 \%$ had bone marrow in- volvement, more common than in non-gastrointestinal MZL lymphomas [16,17]. Therefore, due to its disseminated nature rather than the indolent nature originally believed, MZL can be considered a systemic disease. Alike Topalkara et al. reported a case of conjunctival MZL with amyloid deposit relapsed in the stomach [18], the present case relapsed in the dura resembling the primary dural MZLand was considered relatively rare. Previously, the indolent nature of most MZL cases makes a conservative approach advisable such as treatment by locoregional therapy [1-3]. The MZL patients should be administered chemotherapy and be followed up for an extended period $[19,20]$. After the diagnosis, our patient was given an active radiation treatment and routine chemical therapy. Now he has been followed-up at the outpatient clinic and remained free of specific complaints for 46 months. The patient consented to report this rare case with special manfestation.

The present case suggested that primary cutaneous MZL might present massive amyloid deposition and remote dissemination involving the dura, mimicking a primary dural MZL. The differential diagnosis should be considered to avoid misdiagnosis. Although generally indolent in nature, primary cutaneous MZL has the heterogeneous clinical course. The patients do indeed require long-term regular examinations for possible recurrence and dissemination.

\section{Acknowledgements}

The authors thank Dr. John K. C. Chan of the Pathology Department of Queen Elizabeth Hospital in Hong Kong, China for his help with diagnosis and technical assistance with immunostains. The authors also thank Dr. Dehong Lu and Dr. Yueshan Piao of the Pathology Department of Xuanwu Hospital in Beijing, China for their help with histochemical staining.

\section{Contributions}

M. X. He wrote the paper and organized the Images and patient data. The diagnosis and differentiation were carried out by L. L. Wu, J. J. Wang, C. G. Bai, Y. Wang, and M. H. Zhu. J. J. Wang helped organize and super- vised the writing process. S. M. Zhang was involved in organizing data, evaluating test results and writing the paper.

\section{REFERENCES}

[1] S. H. Swerdlow, E. Campo, N. L. Harris, E. S. Jaffe, et al., "World Health Organization Classification of Tumour, Pathology and Genetics of Tumours of Haematopoietic and Lymphoid Tissues. International Agency for Reseach on Cancer,” IARC Press, Lyon, 2008.

[2] N. J. Senff, E. M. Noordijk, Y. H. Kim, M. Bagot, et al., "European Organization for Research and Treatment of Cancer; International Society for Cutaneous Lymphoma. European Organization for Research and Treatment of Cancer and International Society for Cutaneous Lymphoma Consensus Recommendations for the Management of Cutaneous B-Cell Lymphomas,” Blood, Vol. 112, No. 5, 2008, pp. 1600-1609. doi:10.1182/blood-2008-04-152850

[3] J. H. Cho-Vega, F. Vega, G. Rassidakis and L. J. Medeiros, "Primary Cutaneous Marginal Zone B-Cell Lymphoma," American Journal of Clinical Pathology, Vol. 125, Suppl. 1, 2006, pp. S38-S49.

[4] A. L. Breton, N. Poulalhon, B. Balme, L. Thomas, et al., "Primary Cutaneous Marginal Zone Lymphoma as a Complication of Radiation Therapy: Case Report and Review," Dermatology Online Journal, Vol. 16, No. 12, 2010, p. 6.

[5] S. Pittaluga, L. Bijnens, I. Teodorovic, A. Hagenbeek, et al., "Clinical Analysis of 670 Cases in Two Trials of the European Organization for the Research and Treatment of Cancer Lymphoma Cooperative Group Subtyped According to the Revised European-American Classification of Lymphoid Neoplasms: A Comparison with the Working Formulation,” Blood, Vol. 87, No. 10, 1996, pp. 43584367.

[6] J. D. Gillmore, P. N. Hawkins and M. B. Pepys, “Amyloidosis: A Review of Recent Diagnostic and Therapeutic Developments," British Journal of Haematology, Vol. 99, No. 2, 1997, pp. 245-256.

doi:10.1046/j.1365-2141.1997.303194.x

[7] G. Goteri, R. Ranaldi, S. A. Pileri and I. Bearzi, "Localized Amyloidosis and Gastrointestinal Lymphoma: A Rare Association,” Histopathology, Vol. 32, No. 4, 1998, pp. 348-355. doi:10.1046/j.1365-2559.1998.00409.x

[8] M. Kojima, S. Sugihara, M. Iijima, T. Ono, et al., "Marginal Zone B-Cell Lymphoma of Minor Salivary Gland Representing Tumor-Forming Amyloidosis of the Oral Cavity. A Case Report," Journal of Oral Pathology \& Medicine, Vol. 35, No. 5, 2006, pp. 314-316. doi:10.1111/j.1600-0714.2006.00408.x

[9] T. Satani, T. Yokose, T. Kaburagi, Y. Asato, et al., “Amemiya. Amyloid Deposition in Primary Pulmonary Marginal Zone B-Cell Lymphoma of Mucosa-Associated Lymphoid," Pathology International, Vol. 57, No. 11, 2007, pp. 746-750.

doi:10.1111/j.1440-1827.2007.02164.x 
[10] D. Gupta, V. Shidham, V. Zemba-Palko and A. Keshgegian, "Primary Bilateral Mucosa-Associated Lymphoid Tissue Lymphoma of the Breast with Atypical Ductal Hyperplasia and Localized Amyloidosis. A Case Report and Review of the Literature," Archives of Pathology \& Laboratory Medicine, Vol. 124. No. 8, 2000, pp. 12331236.

[11] P. H. Tu, C. Giannini, A. R. Judkins, J. M. Schwalb, et al., “A. Perry. Clinicopathologic and Genetic Profile of Intracranial Marginal Zone Lymphoma: A Primary LowGrade CNS Lymphoma That Mimics Meningioma,” Journal of Clinical Oncology, Vol. 23, No. 24, 2005, pp. 57185727. doi:10.1200/JCO.2005.17.624

[12] A. C. George,M. Ozsahin, R. Janzer, S. Agassis, et al., "Primary Intracranial Dural Lymphoma of Mucosa-Associated Lymphoid Tissue (MALT) Type: Report of One Case and Review of the Literature," Bull Cancer, Vol. 92, No. 7, 2005, pp. E51-E56.

[13] F. P. Saggioro, B. O. Colli, A. N. Paixão-Becker. G. G. de Rezende, et al., "Primary Low-Grade MALT Lymphoma of the Dura," Histopathology, Vol. 49, No. 3, 2006, pp. 323-326.

[14] S. Bayraktar, A. Stefanovic, N. Montague, J. Davis, et al., "Central Nervous System Manifestations of Marginal Zone B-Cell Lymphoma,” Annals of Hematology, Vol. 89, No. 10, 2010, pp. 1003-1009.

[15] C. Thieblemont, F. Berger, C. Dumontet, I. Moullet, et al., "Mucosa-Associated Lymphoid Tissue Lymphoma Is a
Disseminated Disease in One Third of 158 Patients Analyzed,” Blood, Vol. 95, No. 3, 2000, pp. 802-806.

[16] J. P. de Boer, R. F. Hiddink, M. Raderer, N. Antonini, et al., "Dissemination Patterns in Non-Gastric MALT Lymphoma,” Haematologica, Vol. 93, No. 2, 2008, pp. 201206. doi:10.3324/haematol.11835

[17] C. Suh, J. Huh and J. L. Roh, "Extranodal Marginal Zone B-Cell Lymphoma of Mucosa-Associated Lymphoid Tissue Arising in the Extracranial Head and Neck Region: A High Rate of Dissemination and Disease Recurrence," Oral Oncology, Vol. 44, No. 10, 2008, pp. 949-955. doi:10.1016/j.oraloncology.2007.11.011

[18] A. Topalkara, Y. Ben-Arie-Weintrob, J. A. Ferry and C. S. Foster, "Conjunctival Marginal Zone B-Cell Lymphoma (MALT Lymphoma) with Amyloid and Relapse in the Stomach,” Ocular Immunology and Inflammation, Vol. 15, No. 4, 2007, pp. 347-350. doi:10.1080/09273940701375410

[19] M. Raderer, F. Vorbeck, M. Formanek, C. Osterreicher, et al., "Importance of Extensive Staging in Patients with Mucosa-Associated Lymphoid Tissue (MALT)-Type Lymphoma,” British Journal of Cancer, Vol. 83, No. 4, 2000, pp. 454-457. doi:10.1054/bjoc.2000.1308

[20] M. Raderer, B. Streubel, S. Woehrer, A. Puespoek, et al., "High Relapse Rate in Patients with MALT Lymphoma Warrants Lifelong Follow-Up,” Clinical Cancer Research, Vol. 11, No. 9, 2005, pp. 3349-3352. doi:10.1158/1078-0432.CCR-04-2282 